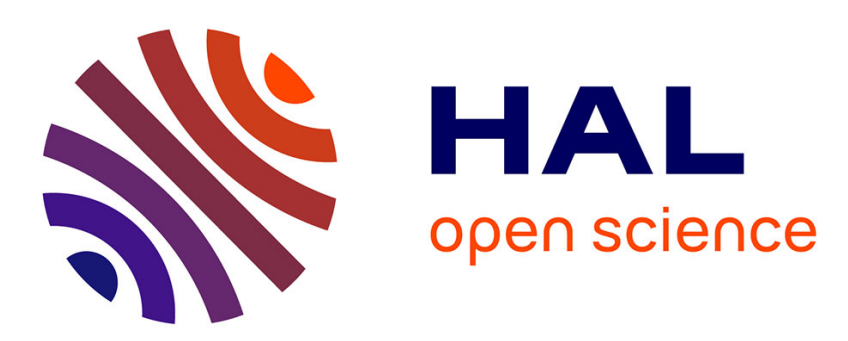

\title{
Stability of bedforms in laminar flows with free surface: from bars to ripples
}

O. Devauchelle, L. Malverti, 'E. Lajeunesse, P.-Y. Lagree, C. Josserand, K.-D. Nguyen Thu-Lam

\section{- To cite this version:}

O. Devauchelle, L. Malverti, 'E. Lajeunesse, P.-Y. Lagree, C. Josserand, et al.. Stability of bedforms in laminar flows with free surface: from bars to ripples. Journal of Fluid Mechanics, 2010, 642, pp.329-348. 10.1017/S0022112009991790 . hal-00555686

\section{HAL Id: hal-00555686 https://hal.science/hal-00555686}

Submitted on 13 Apr 2017

HAL is a multi-disciplinary open access archive for the deposit and dissemination of scientific research documents, whether they are published or not. The documents may come from teaching and research institutions in France or abroad, or from public or private research centers.
L'archive ouverte pluridisciplinaire HAL, est destinée au dépôt et à la diffusion de documents scientifiques de niveau recherche, publiés ou non, émanant des établissements d'enseignement et de recherche français ou étrangers, des laboratoires publics ou privés. 


\title{
Stability of bedforms in laminar flows with free-surface: from bars to ripples
}

\author{
By O. DEVA U C H E L L E ${ }^{1,2}$, L. M A L VER T I ${ }^{1}$, \\ É. L A J E U N ES S E ${ }^{1}$, P. - Y. L A GRÉ E $\mathbf{E}^{3}$, \\ C. J OSSERA N D and K. - D. NGUYEN THU-LA M \\ ${ }^{1}$ Institut de Physique du Globe, 4 place Jussieu, 75252 Paris cedex 05, France \\ ${ }^{2}$ Earth, Atmospheric and Planetary Science, Massachusetts Institute of Technology, \\ 77 Massachusetts Avenue, Cambridge MA 02139-4307, USA \\ ${ }^{3}$ Institut Jean le Rond d'Alembert, Case 161-162, Tour 55-65, 4 Place Jussieu, \\ 75252 Paris Cedex 05, France
}

(Received 9 December 2008)

The present paper is devoted to the formation of sand patterns by laminar flows. It focuses on the rhomboid beach pattern, formed during the backswash. A recent bedload transport model, based on a moving-grains balance, is generalized in three dimensions for viscous flows. It is coupled with the full incompressible Navier-Stokes equations, with a free surface. A linear stability analysis then shows the simultaneous existence of three types of instabilities, namely ripples, bars and longitudinal striations. The comparison of the bar instability characteristics with laboratory rhomboid patterns indicates that the latter could result from the non-linear evolution of unstable bars.

\section{Introduction}

When water flows above a granular layer, the interaction between sediment transport and the fluid motion often leads to instability patterns such as ripples, dunes and bars (see Allen 1982). This is well-known in rivers, where the flow is turbulent, but this is true as well at lower Reynolds number. The most striking example of sediment pattern generated by a laminar flow is the ubiquitous rhomboid beach pattern (see Woodford 1935; Devauchelle et al. 2008). One can observe the formation of these diamond-shaped structures after the swash, when water returns to the sea through a thin film covering the sand. The rhomboid pattern has been found also in geological records (Thompson 1949; Singh 1969), where it may be misinterpreted as the crossing of two successive sets of ripples (Williamson 1887).

Probably due to their likeness, rhomboid beach patterns are generally associated with stationary gravity-waves in super-critical flows. Woodford (1935) first suggested that the sand simply could be marked by such waves, thus neglecting the coupling between the sediment transport and the flow. This theory provides a relationship between the pattern angle $\alpha$ and the Froude number $F r$, namely $\alpha=\arcsin (1 / F r)$. Most subsequent contributions on the subject (Chang \& Simons 1970; Allen 1982) are based on the same idea. However, Devauchelle et al. (2008) showed experimentally that Woodford's law strongly overpredicts the pattern angle. Ikeda (1983) proposed that the rhomboid pattern might result from the association of antidunes and longitudinal striations. This idea will be addressed in the present paper (see section 3.3).

After their own experimental observations, Karcz \& Kersey (1980) concluded that 

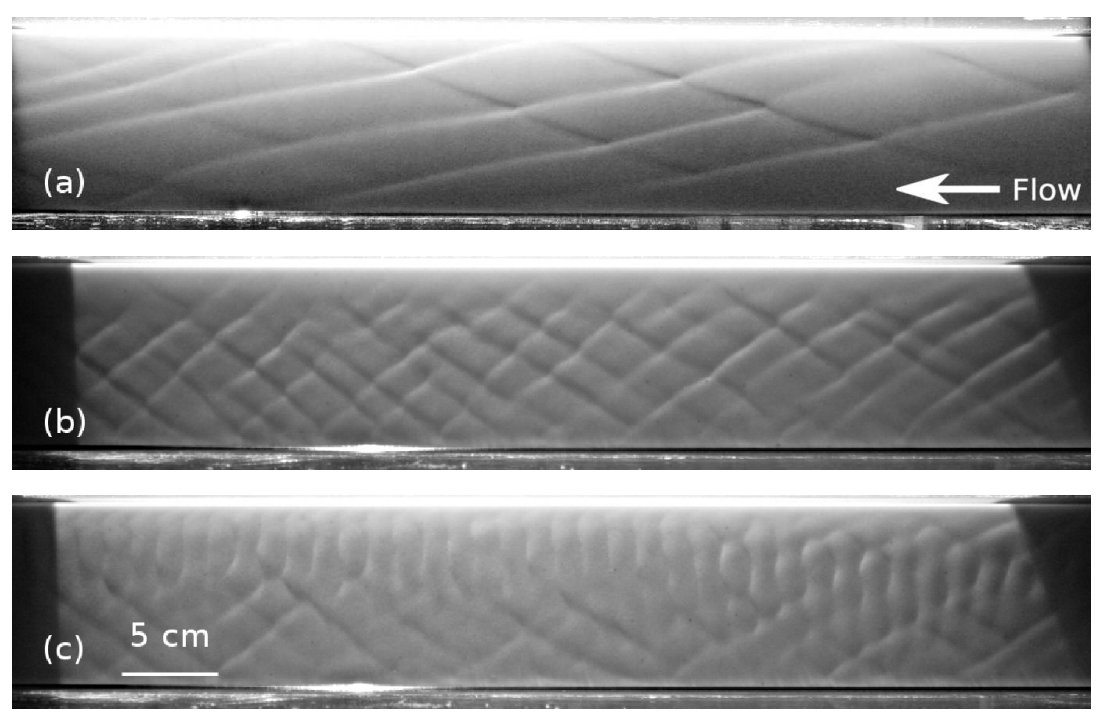

FIGURE 1. Various bedforms observed on the granular bed of a a laminar flume (Devauchelle et al. 2008). The flow is from right to left. The pattern, observed through the water surface, is enhanced by skimming light. The angle and wavelength of the rhomboid pattern varies with the experimental parameters (picture (a) and (b)). Under certain conditions, a rhomboid pattern may be associated to ripples (picture (c)). The width of the flume is $10 \mathrm{~cm}$. (a) Large rhomboid pattern $(F r=1.76, S=0.03, B o=1.31$ and $S h=0.616)$. (b) Small rhomboid pattern $(F r=0.95, S=0.015, B o=3.25$ and $S h=0.485)$. (c) Rhomboid pattern mixed with ripples $(F r=1.01, S=0.015, B o=3.50$ and $S h=0.504)$. The definitions of the parameters are provided in section 3.2 .

a homogeneous rhomboid pattern forms spontaneously over an initially flat bed, thus suggesting an absolute instability. In a first attempt to perform the stability analysis of a granular bed submitted to erosion by a laminar film, Devauchelle et al. (2008) used the Saint-Venant approximation to represent the water flow. These equations, written for turbulent flows, are known to produce rhomboid patterns under certain conditions (see Defina 2003; Hall 2006). Since the equations for laminar films are very similar to their turbulent counterparts, the shallow-water theory also predicts that a bar instability should develop. In turn, this instability could lead to diamond-shaped structures after saturation, due to non-linear effects. Consequently, the rhomboid beach pattern can be related to alternate bars in rivers (Callander 1969). However, the Saint-Venant equations fail to predict the pattern angle better than the Woodford's law does (although they underpredict it instead). The results are even worse regarding the wavelength, but the theory at least qualitatively agrees with the observation of Karcz \& Kersey (1980) that a uniform wavelength spontaneously emerges.

The main flaw of the Saint-Venant equations regarding bedforms is that they cannot represent the phase lag between a bed perturbation and the velocity profile above it. When establishing the Saint Venant equations, one supposes that the profile remains of same shape (namely the Poiseuille parabolic shape). But due to the acceleration over the crest of the ripple, the velocity profile is changed near the bed. This effect is responsible for the formation of ripples (see Kennedy 1963; Charru \& Mouilleron-Arnould 2002; Lagrée 2003), which appeared regularly during the experiments of Devauchelle et al. (2008) (see also figure 1). This observation suggests that the shallow-water approximation is inappropriate under such experimental conditions, and therefore should be replaced by 


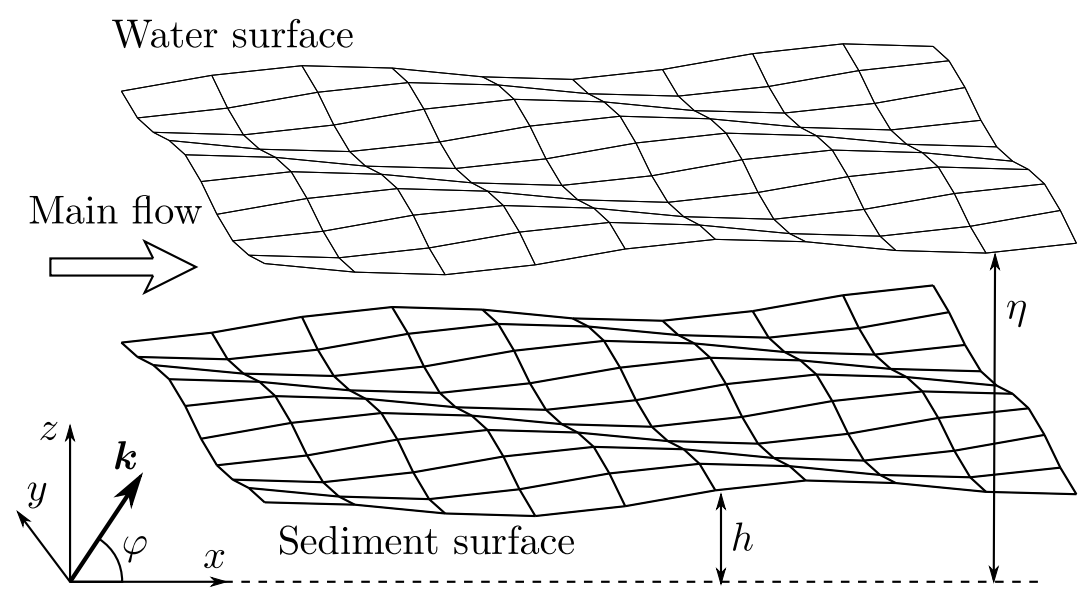

Figure 2. Schematic of the system and associated notations. A film of fluid with a free surface flows over a granular layer. The main flow direction is denoted $x$, and its transverse and vertical counterparts are $y$ and $z$ respectively. The sediment surface is represented by $h(x, y, t)$, and the fluid free surface by $\eta(x, y, t)$. The whole frame is tilted with respect to gravity in the $x$ direction, so that the gravity acceleration $\boldsymbol{g}$ has components $\left(g_{x}, g_{z}, 0\right)$ with $-g_{x} / g_{z}=\tan S$, thus defining the slope $S$. In section 3 , the stability of a perturbation with wave vector $\boldsymbol{k}$ is analyzed. For the sake of simplicity, we do not impose boundaries in the $x$ and $y$ directions.

the full Navier-Stokes equations in three dimensions. It is the subject of the present contribution.

Coupling the water flow to the bed evolution requires a sediment transport model. As long as bedload is the main transport mode, the grains motion is driven by the water shear stress on the sediment surface only. Transport laws are generally written as functions of the Shields parameter, which compares the tangential shear applied to the bed to its normal counterparts (usually the weight of the upper grains layer, see Shields (1936)). However, in order to avoid the instability of short-wavelength bedforms, one has to introduce a saturation mechanism. This can be achieved by taking into account either the bed slope effect or the time required for the particles flux to reach equilibrium (see Lagrée 2000; Charru et al. 2004; Charru \& Hinch 2006; Charru 2006). The present paper generalizes the model proposed by Charru (2006) to three dimensions, thus retaining both saturation mechanisms. As the full Navier-Stokes equations are employed, no strong approximation about the flow remains. For this reason, the rhomboid pattern can provide an experimental test for sediment transport models.

\section{General model}

We aim here to study the linear stability of an initially flat sediment layer, as a laminar and steady film of water flows over it. For simplicity, we assume an infinite system both in the direction $x$ of the main slope and in the transverse direction $y$ (see figure 2). This is consistent with the usual configuration of sand beaches where rhomboid patterns form, where the horizontal dimensions (say a few tens of meter) are far larger than the typical depth $D$ of the backswash film (a few millimeters). It may however be a crude representation of the experiments of Devauchelle et al. (2008), performed in a $10 \mathrm{~cm}$-wide channel. 


\subsection{Water flow}

The flow in the bulk is given by the stationary and incompressible Navier-Stokes equations in three dimensions,

$$
u_{k} \partial_{k} u_{i}=-\frac{1}{\rho} \partial_{i} p+g_{i}+\nu \partial_{k k} u_{i}, \quad \partial_{k} u_{k}=0
$$

where $\boldsymbol{u}, p$ and $\nu$ are respectively the velocity, pressure and viscosity of water, and $\boldsymbol{g}$ is the acceleration of gravity. The use of stationary flow equations is a common hypothesis in geomorphology, which relies on the idea that the sediment transport time scale is much larger than the flow one (see Colombini \& Stocchino 2005). If we neglect both the velocity of the upper layers of grains and the velocity of water through the same layert, we may impose the classical no-slip boundary condition at the sediment bed surface:

$$
u_{i}=0 \quad \text { at } \quad z=h
$$

where $z$ is the vertical direction $\ddagger$, and $h$ is the thickness of the sediment layer. At the free surface, which elevation is denoted by $\eta$, both a kinematic and a dynamical boundary conditions are imposed:

$$
u_{z}=u_{x} \partial_{x} \eta+u_{y} \partial_{y} \eta \quad \text { at } \quad z=\eta, \quad \sigma_{i k} n_{k}=\gamma \kappa n_{i} \quad \text { at } \quad z=\eta
$$

where $\gamma$ and $\boldsymbol{n}$ are respectively the surface tension of the fluid, and the unit vector normal to the surface and pointing outwards. The free-surface curvature is referred to as $\kappa$, which is linked to the surface elevation through the relation

$$
\kappa=\frac{\partial_{x x} \eta}{\left(1+\left(\partial_{x} \eta\right)^{2}\right)^{3 / 2}}+\frac{\partial_{y y} \eta}{\left(1+\left(\partial_{y} \eta\right)^{2}\right)^{3 / 2}} .
$$

The stress tensor is denoted by $\boldsymbol{\sigma}$, whereas $\boldsymbol{\tau}$ is its viscous component only:

$$
\sigma_{i k}=\tau_{i k}-p \delta_{i k}, \quad \tau_{i k}=\rho \nu\left(\partial_{i} u_{k}+\partial_{k} u_{i}\right)
$$

The flow model presented above is rather general for gravity-driven and laminar films. The stability of such films, without any sediment transport, has been intensively studied, and they were proven generally instable with respect to long-wavelength perturbations (Yih 1963), leading to the formation of Kapitza waves (Kapitza 1948). Such films present a wide variety of instable waves, stationary or not. Indeed, Kapitza waves are linearly unstable in two dimensions, but one can expect three-dimensional structures to be also linearly unstable (Lin \& Chen 1997). One could claim that such purely hydrodynamic waves generates the rhomboid pattern, in an extended version of the theory of Woodford (1935). However, the present flow model is stationary, and thus cannot describe any purely-fluid instability. Only in the next section on sediment transport will time enter the picture. Consequently, the stability analysis of the present paper can only account for a fully coupled sediment-flow interaction. During their experiments, Devauchelle et al. (2008) observed both stationary and traveling hydraulic waves. None of them seemed to interact much with the sediment patterns, which growed at a much slower time scale and with different angle than these waves.

$\dagger$ The two assumptions are reasonable in the experiments. This can be evaluated by comparing the measured flow depth to Nußelt's prediction for a given water discharge.

$\ddagger$ More precisely, $z$ is the direction perpendicular to the $(x, y)$-plane, which is slightly tilted in the $x$ direction. 


\subsection{Sediment transport}

A sediment transport model based on a grains balance in the moving sediment layer was first proposed by Lagrée (2000). It has been used for liquid flows (Valance \& Langlois 2005) as well as for air flows (Valance \& Rioual 1999; Sauermann et al. 2001; Andreotti et al. 2002; Hersen 2004), and widely developed for viscous flows by Charru et al. (2004). The latter authors were able to measure the individual grains velocities during bedload transport. Their model for averaged transport is thus supported experimentally at each step of its derivation. The last version of this theory has been adapted to gently sloping beds (Charru \& Hinch 2006; Charru 2006). Values are provided for the empirical coefficients of the theory, that allow to fit the experimental data of Coleman \& Eling (2000). In the present section, we aim to generalize this model in three dimensions, by following the reasoning of Charru (2006). We will end up with the remarkable result that no additional coefficient is required to do so.

\subsubsection{Moving particles conservation equation}

Let us denote by $n$ the density of transported particles per unit surface (as much as possible, we use the notations of Charru (2006) in the following). Then the grains balance in the bedload layer reads

$$
\frac{\partial n}{\partial t}=\dot{n}_{e}-\dot{n}_{d}-\frac{\partial q_{x}}{\partial x}-\frac{\partial q_{y}}{\partial y}
$$

where $\dot{n}_{e}, \dot{n}_{d}$, and $\boldsymbol{q}$ denote respectively the rate of grains erosion from the fixed layer, the deposition rate to the fixed layer and the horizontal grains flux. At first order, and for small bed slopes, this equation does not differ from the flat-bed case, even though the direction of $\boldsymbol{q}$ is no longer in the tilted $(x, y)$-plane†.

\subsubsection{Particles deposition rate}

The grains deposition rate due to gravity-induced sedimentation does not change when extending the model to three dimensions. Provided the suspended particles concentration $n$ remains small enough, each particle behaves as if it were isolated. Due to gravity, it falls down at the Stokes settling velocity $U_{s}$, defined by

$$
U_{s}=\frac{\left(\rho_{s}-\rho\right)\|\boldsymbol{g}\| d_{s}^{2}}{18 \rho \nu}
$$

where $d_{s}$ is the typical diameter of a sediment grain. Every suspended particle experiences sedimentation. The associated flux is thus proportional to the number of suspended particles $n$ :

$$
\dot{n}_{d}=c_{d} \frac{U_{s}}{d_{s}} n,
$$

where $c_{d}$ is an empirical constant, set to $1 / 15$ after Charru (2006).

\subsubsection{Generalized Shields parameter}

Originally, the Shields parameter was designed to compare the weight of a sediment particle (corrected by its buoyancy) to the shear stress exerted by the fluid (Shields 1936), that is:

$$
S h=\frac{\rho\left\|\boldsymbol{\tau}_{h}\right\|}{\left(\rho_{s}-\rho\right)\|\boldsymbol{g}\| d_{s}},
$$

$\dagger$ For bedload on an arbitrary sediment surface, the flux $\boldsymbol{q}$ is tangent to the surface. In the Exner equation, only its horizontal components $q_{x}$ and $q_{y}$ must be considered. 
where $\rho_{s}$ is the density of the sediment. The tensor $\boldsymbol{\tau}_{h}$ represents the horizontal viscous stress. Now, if the local tilt of the sediment bed is to be considered, one has to compare the normal stress component to the tangential one instead. It is the purpose of the following.

If $\boldsymbol{n}^{b}$ is the unit vector normal to the sediment surface, then the viscous force exerted on a surface of small area $\epsilon$ of the sediment surface reads

$$
f_{i}^{\nu}=\tau_{i k} n_{k}^{b} \epsilon .
$$

If the pressure field is uniform at the grain scale, gravity is the only other force acting on the moving grains layer, the thickness of which is denoted by $c_{g} d_{s}$, where $c_{g}$ is assumed to be constant $\left(c_{g} \approx 0.108\right)$ :

$$
f_{i}^{g}=\left(\rho_{s}-\rho\right) g_{i} \epsilon c_{g} d_{s} .
$$

The total force $\boldsymbol{f}=\boldsymbol{f}^{\nu}+\boldsymbol{f}^{g}$ may then be separated into its normal and tangential components:

$$
f^{n}=f_{k} n_{k}^{b}, \quad \boldsymbol{f}^{t}=\boldsymbol{f}-f^{n} \boldsymbol{n}^{b} .
$$

Note that $f^{n}$ is a negative quantity. In this frame, the natural generalization of Shields parameter consists in writing

$$
\theta=-\frac{\left\|\boldsymbol{f}^{t}\right\|}{f^{n}} .
$$

Through this definition of the Shields parameter, one recovers the two-dimensional theory of Charru (2006) when considering a two-dimensional problem in the $(x, z)$ plane. In that sense, the present model is a generalization of the latter. The hypothesis that $\theta$ only controls the intensity of the sediment flux relies on two basic assumptions:

- The effect of slope is isotropic with respect to $x$ and $y$;

- The thickness $c_{g} d_{s}$ of the moving grains layer is constant.

The first assumption is reasonable in general, whereas we are not aware of any direct evaluation of the moving-grains layer thickness. Charru (2006) makes the second assumption when stating that $c_{g}$ is a constant. As long as the bed is perfectly flat, the moving-grains layer thickness itself can be a function of the Shields parameter as usually defined (that is by equation (2.9)), and thus the intensity of the transport is a function of $S h$ only. As soon as some slope is to be considered, it enters the picture as a new independent parameter, and the above reasoning should not hold. However, Charru (2006) showed that treating $c_{g}$ as a constant can lead to good results. We do the same here.

Finally, we only add the isotropic transport assumption to the model of Charru (2006). It allows us to introduce no additional free parameter.

\subsubsection{Particle erosion rate}

As for the deposition rate, the erosion rate $\dot{n}_{e}$ can be expressed exactly in the same way as in Charru (2006). We usually consider that below a threshold value of the Shields parameter denoted by $\theta_{t}$, no particle can be suspended. Above this value, the simplest model consists in assuming that the number of particles suspended by unit time and surface is proportional to the excess Shields parameter, that is, proportional to $S h-\theta_{t}$. In the present case, the same idea can be applied to the generalized Shields parameter $\theta$. We thus assume that $\dot{n}_{e}$ is proportional to $c_{g} \theta-\theta_{t}$. The constant $c_{g}$ appears only due to our definition of $\theta$. With these notations, the threshold value remains the one proposed by Charru (2006), namely $\theta_{t} \approx 0.091$. The choice of the dimensional pre-factors then fixes the empirical constant $c_{e}$ :

$$
\dot{n}_{e}=\frac{18 c_{e} U_{s}}{d_{s}^{3}}\left(c_{g} \theta-\theta_{t}\right),
$$


Charru (2006) fixes $c_{e}$ to 0.0017 , but this value has no impact on the pattern shape. In the above relation, the slope influence on the tangential shear stress is embedded into the definition of $\theta$.

\subsubsection{Particle horizontal flux}

While they are suspended, the grains are transported by the water flow, thus generating a horizontal sediment flux $\boldsymbol{q}$. Charru et al. (2004) showed experimentally that, in a good approximation, the average particle velocity is proportional to the vertical gradient of the horizontal velocity, times the average flight height. The flux is then obtained by multiplying this average velocity with the suspended particles concentration $n$ :

$$
q_{x}=n c_{u} d_{s} \frac{\partial u_{x}}{\partial z}, \quad q_{y}=n c_{u} d_{s} \frac{\partial u_{y}}{\partial z},
$$

where $c_{u} \approx 0.1$ is the third and last empirical parameter of the sediment transport law. This hypothesis can be understood as a simplified version of Bagnold's model of sediment transport by rivers (Bagnold 1977).

\subsubsection{Exner equation}

The above system, from equation (2.6) to equation (2.15) could be solved without further assumption. However, to remain consistent with the stationary flow model (see section 2.1), we must drop the time derivative in the sediment balance equation (2.6). Thus, the particle flow is considered stationary as well and we end up with the well-known Exner equation (Exner 1925):

$$
C \partial_{t} h=-\frac{\pi d_{s}^{3}}{6}\left(\partial_{x} q_{x}+\partial_{y} q_{y}\right),
$$

where $C$ is the bed compactness. One should bear in mind that the above equation, a balance for the immobile grains, does not replace the balance (2.6) for mobile grains. The latter is required to determine the moving particle density $n$, even under the quasistationary approximation. In brief, one can determine the concentration of suspended particles using (2.6) with the quasi-stationary approximation $\partial n / \partial t=0$. Then, the equation (2.16) controls the topography evolution.

The Exner equation terminates the definition of our sediment transport model, and the following section is devoted to the stability analysis of a flat bed within this framework.

\section{Stability analysis}

The present section aims to demonstrate that, under the experimental conditions where rhomboid patterns where observed by Devauchelle et al. (2008), the model proposed above is unstable. As stated in the introduction of this paper, it is our claim that the rhomboid pattern can maintain its basic features (angle and wavelength) during its development. In other words, we believe (but do not prove) that the non-linear effects allowing for the bed perturbations to saturate do not modify significantly theses features. If this is true, the results of the stability analysis can be compared to measurements (see section 4), even though the latter concerns fully developed bedforms rather than infinitesimal perturbations.

\subsection{Base state and dimensions}

The simplest base state for our system consists in a flat (but tilted) sediment bed, over which flows a uniform Nußelt film of thickness $D$. This roughly corresponds both to the 
experiments (a rake was drawn over the granular bed before each run), and to the natural initial condition on beaches (each swash event erases the remaining patterns before the backswash).

The Nußelt film above the bed reads

$$
u_{x}^{0}=\frac{g_{x}}{\nu}\left(z D-\frac{z^{2}}{2}\right), \quad p^{0}=-\rho g_{z}(D-z),
$$

where the 0 superscript refers to the base state. The water velocity vanishes in both the $y$ and the $z$ directions. If $\mathcal{U}, \mathcal{D}$ and $\mathcal{P}$ respectively refer to the typical scales of the water velocity, depth and pressure, we define

$$
\mathcal{U}=\frac{g_{x}}{3 \nu} D^{2}, \quad \mathcal{D}=D, \quad \mathcal{P}=-\rho g_{z} D
$$

The non-dimensional base state then reads

$$
u_{x}^{0}=\mathcal{U} U\left(\frac{z}{D}\right), \quad p^{0}=\mathcal{P} P\left(\frac{z}{D}\right)
$$

with

$$
U(\tilde{z})=\frac{3 \tilde{z}}{2}(2-\tilde{z}), \quad P(\tilde{z})=(1-\tilde{z})
$$

The tilde here denotes a non-dimensional height. In the following, all quantities are nondimensional, and the tilde will be dropped for clarity.

\subsection{Perturbations}

Let $f$ refers to any quantity of interest for our problem. Decomposing it into a base-state component $F$ and a sine-wave perturbation of complex amplitude $f^{*}(z)$ leads to

$$
f(x, y, z, t)=F(z)+f^{*}(z) e^{i(k \cos \varphi x+k \sin \varphi y-\omega t)},
$$

where $k, \varphi$ and $\omega$ are respectively the wave-vector norm, its angle with respect to the $x$ axis, and the pulsation of the perturbation. The non-dimensional wave vector is normalized by $\mathcal{D}$. In the same fashion, the time-scale $\mathcal{T}$ for the pulsation $\omega$ is given by the Exner equation (2.16):

$$
\mathcal{T}=\frac{6 C \mathcal{D}^{2}}{\pi l_{d} c_{d} U_{s} d_{s}^{2} N}
$$

where $l_{d}$ and $N$ are respectively the deposition length defined by Charru (2006), and the suspended concentration of the base flow:

$$
l_{d}=\frac{3 c_{u} \mathcal{U} d_{s}^{2}}{c_{d} U_{s} \mathcal{D}}, \quad N=\frac{18 c_{e}}{c_{d} d_{s}^{2}}\left(c_{g} \Theta-\theta_{t}\right) .
$$

The deposition length, which corresponds to the order of magnitude of the average flight length of a particle, introduces a space gap between the sediment flux and the shear stress that generates it. This delay stabilizes the perturbations at short wavelengths (see Charru \& Mouilleron-Arnould 2002; Andreotti et al. 2002; Lagrée 2003).

\subsubsection{Linearized flow equations}

For small perturbations, the momentum- and mass-conservation equations for the fluid (2.1) read

$$
\begin{gathered}
F r^{2}\left(i U k \cos \varphi u_{x}+U^{\prime} u_{z}\right)=-i k \cos \varphi p+\frac{S}{3}\left(u_{x}^{\prime \prime}-k^{2} u_{x}\right) \\
F r^{2} i U k \cos \varphi u_{y}=-i k \sin \varphi p+\frac{S}{3}\left(u_{y}^{\prime \prime}-k^{2} u_{y}\right)
\end{gathered}
$$




$$
\begin{gathered}
\text { Journal of Fluid Mechanics } \\
\operatorname{Fr}^{2} i U k \cos \varphi u_{z}=-p^{\prime}+\frac{S}{3}\left(u_{z}^{\prime \prime}-k^{2} u_{z}\right), \\
u_{z}^{\prime}+i k\left(\cos \varphi u_{x}+\sin \varphi u_{y}\right)=0
\end{gathered}
$$

where $F r=\mathcal{U} / \sqrt{-g_{z} \mathcal{D}}$ is the Froude number. The prime denotes derivation with respect to the non-dimensional height $z$. The no-slip boundary condition (2.2) at the bed surface in turn reads

$$
u_{x}+3 h=0, \quad u_{y}=0, \quad u_{z}=0 .
$$

At the free surface, the kinematic boundary condition imposes

$$
u_{z}=\frac{3}{2} i k \cos \varphi \eta,
$$

whereas the dynamic boundary condition reads

$$
-3 \eta+u_{x}^{\prime}+i k \cos \varphi u_{z}=0, \quad i k \sin \varphi u_{z}+u_{y}^{\prime}=0, \quad \eta-p+\frac{2}{3} S u_{z}^{\prime}=-\frac{k^{2}}{B o} \eta,
$$

where $B o=-g_{z} \rho \mathcal{D}^{2} / \gamma$ is the Bond number.

\subsubsection{Linearized sediment transport equations}

Let us define $N$ as the concentration scale for $n$. Then, combining this definition with the linearized grains balance relation for stationary sediment transport (2.6) leads to

$$
\frac{\Theta}{\Theta-\theta_{t} / c_{g}} \theta^{*}-n^{*}-\frac{l_{d}}{3 \mathcal{D}} i k\left(3 n^{*}+k \cos \varphi u_{x}^{* \prime}+\sin \varphi u_{y}^{* \prime}\right)=0,
$$

where the generalized Shields parameter for the base state $\Theta$ reads

$$
\Theta=\frac{S h}{c_{g}}+S=\frac{3 \mathcal{U} \nu \rho}{c_{g} d\left|g_{z}\right| \mathcal{D}\left(\rho_{s}-\rho\right)}+S .
$$

The perturbation $\theta^{*}$ for the Shields parameter is given by equation (2.13):

$$
\begin{aligned}
\theta^{*}=\frac{1}{3}\left(2 \tilde{\theta}^{2}\left(u_{z}^{* \prime}-3 i h^{*} k \cos \varphi\right)-\right. & 3 i h^{*} k \cos \varphi\left(1+S^{2}\right)+ \\
& \left.\frac{S h}{c_{g}}\left(u_{x}^{* \prime}+2 S u_{z}^{* \prime}-3 h^{*}(1+3 i k \cos \varphi S)\right)\right) .
\end{aligned}
$$

Finally, the Exner equation (2.16) allows to close the linear system, as did equation (2.16) for the complete model:

$$
\omega h^{*}=k\left(3 n^{*}+\cos \varphi u_{x}^{* \prime}+\sin \varphi u_{y}^{* \prime}\right) .
$$

\subsection{General features of the bed stability}

The system of ordinary differential equations (3.8), (3.9), (3.10) and (3.11) is linear. For any wave vector $\boldsymbol{k}$, it can thus be solved numerically by means of the linear shooting method to fit the boundary conditions (3.12), (3.13) and (3.14). Likewise, once the sediment transport law is defined by its empirical parameters $\theta_{t}, c_{u} / c_{d}$ and $c_{g}$ (respectively $0.091,0.9$ and 0.108 in the present case), one can derive the complex pulsation $\omega$ from equations (3.15), (3.17) and (3.18). Finally, for a given set of experimental parameters $S, F r, B o$ and $S h$, the dispersion relation of our system is obtained.

\subsubsection{Striations, ripples and bars}

The dispersion relation for the bed perturbation provides informations about both the velocity of sand waves and their stability. In the present paper, we will focus on 


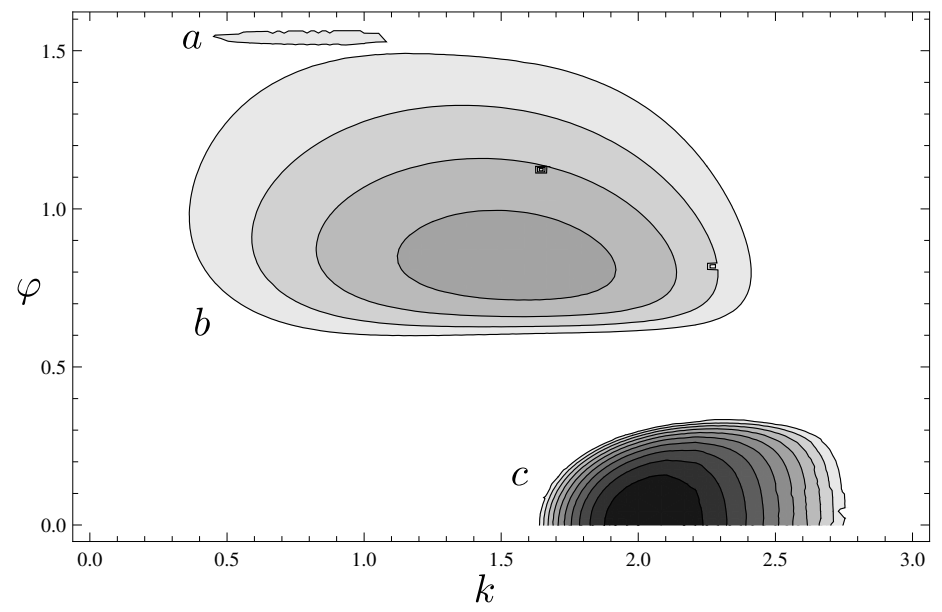

FiguRE 3. Growth rate $\operatorname{Im}(\omega)$ of the tree-dimensional instabilities of a granular bed submitted to erosion by a laminar flow, as a function of the wave-vector norm $k$, and angle $\varphi$ with respect to the $x$ direction. The blank domain corresponds to negative values of the growth rate, that is, stable modes. In this typical example, three types of instabilities can develop. (a) longitudinal striation (see Ikeda 1983; Karcz \& Kersey 1980), which maximum growth rate lies at angle close to $\pi / 2$; (b) bar instability, which can occur at any value of the angle $\varphi$; (c) ripple instability, which crests are perpendicular to the main flow direction, that is, $\varphi=0$. The bar instability is probably responsible for the initiation of rhomboid patterns. In this example, the parameters have values $F r=0.90, S=0.015, B o=2.77$ and $S h=0.448$. Each contour line corresponds to an increment of 4.8 for the growth rate. The approximate values of the three maxima of this figure are (a) 7, (b) 22 and (c) 53 .

stability issues, since the associated predictions (pattern angle and wavelength) are easily measured experimentally.

The growth rate of a typical example is plotted on figure 3. The existence of three distinct maxima is the most striking feature of this dispersion relation. This is not always the case. For other values of the parameters, any association of these three types of maxima is possible, which can makes the distinction uneasy. Hereafter, we will refer to the following denominations:

- Longitudinal striations correspond to maximum nearest to $\varphi=\pi / 2$ (the mode (a) on figure 3);

- Ripples correspond to a growth rate maximum lying on the $k$ axis, that is, for $\varphi=0$ (the mode (c) on figure 3);

- Bars correspond to any other maximum (the mode (b) on figure 3).

The value of the angle $\varphi$ usually allows to discriminate between bars and longitudinal striations, the latter being always unstable (in a reasonable range of parameter values).

Longitudinal striations where observed by Karcz \& Kersey (1980) in experimental laminar flumes, sometimes coexisting with rhomboid patterns. Such bedforms are named ridges by these authors. They are also referred to as current lineations and associated to sublayer streaks (Colombini \& Parker 1995; Jackson 2006) or Görtler vortex (Langlois 2005). In turbulent flows, Ikeda (1983) suggests that longitudinal striations could mix with ripples to form a rhomboid pattern. In laminar flows, his views are not supported by the analysis developed in the present paper (see section 3.3.2). Ridges where also produced during the experiment of Devauchelle et al. (2008), but they always remained faint, and usually disappeared a few seconds after the run was started. Although we are not aware of any previous contribution on the stability analysis of such longitudinal 

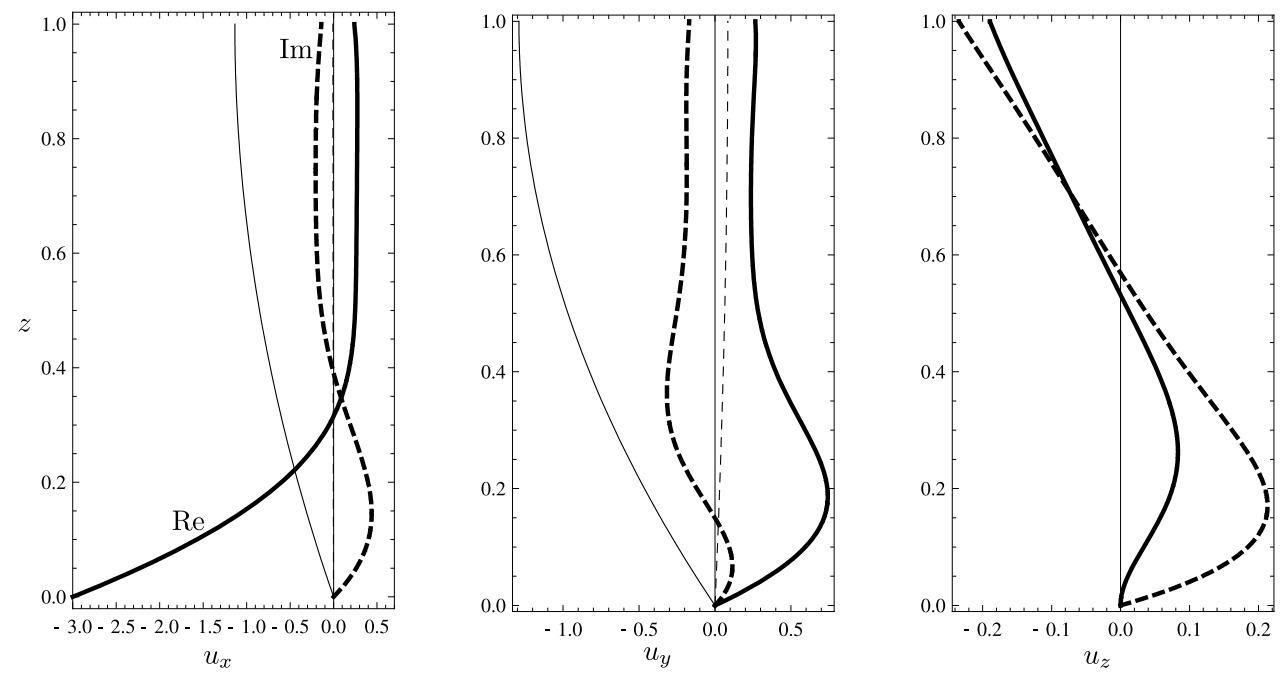

FIGURE 4. Complex velocities field for the bar instability (thick lines). The solid line represents the real part of the velocity perturbation, whereas the dashed line represents its imaginary part. The corresponding Saint-Venant velocities are represented by thin lines for comparison. The shape of the perturbation in the full three dimensional case differs significantly from the Nußelt parabola. These velocity profiles correspond to the maximum for the bar instability of figure 3 , that is, $k=1.5$ and $\varphi=0.85$.

patterns in laminar flows, we postpone their systematic study to future work, since the present contribution focuses on rhomboid patterns.

The formation of sand ripples has received much attention, as an ubiquitous geomorphological pattern, since the early works of Exner (1925), Kennedy (1963) or Reynolds (1965). The mechanism leading to the ripple instability is now clearly established. It is similar in laminar (see Charru \& Mouilleron-Arnould 2002; Lagrée 2003) and turbulent flows (see Colombini 2004; Elbelrhiti et al. 2005). Its basics can be reduced to the $(x, z)$ plane, that is, in the main flow and vertical directions. On the windward side of a bump, the water inertia draws the flow lines closer together, and the velocity increases. The reverse occurs on the leeward side. The shear stress being a combined effect of the velocity variation by the streamlines thinning, the net effect is that skin friction is larger on the windward side than on the leeward side. Now, viscosity induces a slight asymmetry of the flow, which makes the skin friction extremal just before the crest (Lagrée 2003). The Exner equation (2.16) then indicates that deposition occurs just after the bump, thus growing the perturbation and moving it forwards. This process being two-dimensional, it can only create invariant structures in the transverse direction. The term ripples generally implies this $y$ invariance, at least during the initiation stage. A second characteristic of the ripple-formation mechanism is that the velocity profile is locally strongly accelerated near the bed and then differs strongly from the Poiseuille flow. This cannot be represented by the Saint-Venant equations and needs some triple deck boundary layer theories to be asymptotically modeled (Kouakou \& Lagrée 2005). It explains why any bed-stability analysis performed in the frame the shallow-water equations predicts stable ripples at any wavelength (Devauchelle et al. 2008). Finally, ripples can grow in deep water or in a pipe, that is, without any free surface (see Kuru et al. 1995).

The three criteria listed above allow one to distinguish theoretically between ripples and sand bars. Contrary to ripples, bars require the influence of a free surface, and can be 
modeled by means of the Saint-Venant equations. Their wave vector is usually inclined with respect to the main flow direction (see figure 3), and thus cannot be represented in the $(x, z)$-plane only. To our knowledge, the first mathematical derivation of this instability is due to Callander (1969), and was soon associated to river meandering, although this point remains controversial (Ikeda et al. 1981; Blondeaux \& Seminara 1985). The most striking occurrence of the bar instability in Nature are the alternate bars in rivers (Knaapen \& Hulscher 2003), which results from the crossing of two bars instabilities of angle $\varphi$ and $-\varphi$. It has been demonstrated, both experimentally (Lajeunesse et al. 2008) and theoretically (Devauchelle et al. 2007), that laminar flumes are also prone similar bars development. Langlois \& Valance (2005) performed a linear stability analysis comparable to the present one. However, their analysis was designed for a pipe flow, and thus did not include a free-surface. As a consequence, their results are very similar to ours as far as the ripple instability is concerned, but do not include any bar instability.

Bars and ripples may be distinguished as two limiting cases of sediment-flows instability. However, in the range of parameters explored by Devauchelle et al. (2008) to study rhomboid patterns, the two are mixed in a bar-type instability which presents some ripples features. The figure 4 illustrates this point. The velocity field above a wavy bed corresponding to the bar instability is obviously not parabolic, that is, is not proportional to a Nußelt film. Instead, inertia controls the velocity profile as well as viscosity. This explains why the Saint-Venant equations fail to predict quantitatively the angle of the rhomboid pattern. The linear interaction between bars and ripples is discussed in the following section.

\subsubsection{Transition from bars to ripples}

When the Froude number is high enough, bars present a large wavelength, and they are very elongated in the flow direction (the angle $\varphi$ is close to $\pi / 2$ ). They are thus far enough from the ripple instability not to interact with it. In that case, they can be roughly modeled by the Saint-Venant equations. The opening angle predicted by these equations approaches the experimental bedforms one as Fr increases (Devauchelle et al. 2008). However, the rhomboid pattern wavelength is largely overpredicted by a shallow-water model, even for large Froude numbers.

The present model provides an explanation for the failure of the Saint-Venant equations as the Froude number decreases. The figure 5 shows the evolution of bar and ripple instabilities with respect to the Froude number. Initially $(F r=0.90$ on figure 3 and $F r=0.86$ on figure 5 ), the two instabilities are clearly distinct. Now, if the Froude number is reduced to 0.7 and then to 0.5 , the growth-rate maximum corresponding to the bar instability slides towards smaller $\varphi$ values, while the ripples maximum disappears. For even lower Froude numbers, the maximum related to the bar instability has reached the $k$-axis, and thus has become a ripple instability after the definitions of section 3.3.1. This transition occurs sharply with respect to the Froude number, and can be generalized to other values of the other parameters (provided they lie in the range where both instabilities exist). During this process, the maximum corresponding to the longitudinal striations does not evolve significantly.

The theory proposed by Ikeda (1983) to describe the formation of rhomboid patterns in turbulent flumes must probably be rejected, at least in the laminar case. Indeed, the growth rate maximum associated to longitudinal striations seems not to play an essential role in the angle transition of the rhomboid pattern. On figures 3 and 5 , it seems to remain at the same place in the $(k, \varphi)$-plane, while the bar and ripple instabilities interact. It would thus seem rather unnatural to interpret the bar instability as the superposition of ripples and longitudinal striations. 

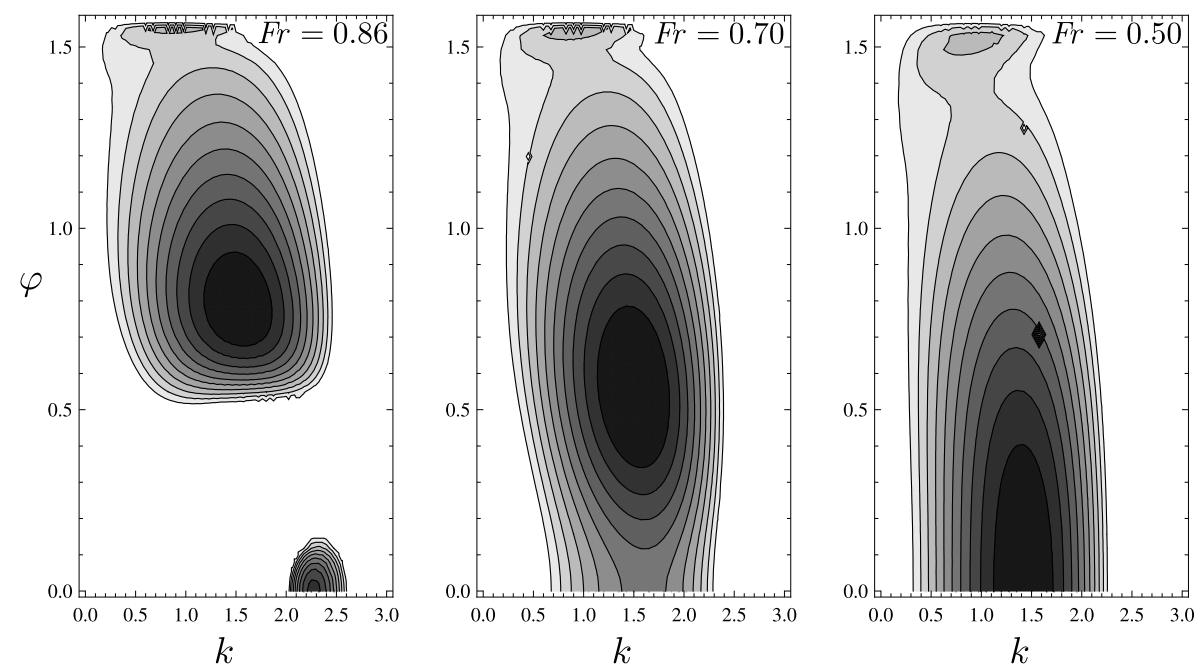

Figure 5. Transition from bars to ripples as the Froude number decreases. The longitudinal striations seem not to be significantly affected by this transition. The other parameters are fixed to $S=0.015, B o=2.77$ and $S h=0.448$, as for figure 3 . The transition induces a decrease in the angle of the most instable mode. This behavior was qualitatively predicted by Woodford (1935), without referring to this interaction between bars and ripples. Each contour line corresponds to a increment of 2 for the growth rate.

This transition from bars to ripples is able to explain the angle transition of the rhomboid pattern occurring at low Froude numbers. The following section is devoted to the experimental evaluation of this hypothesis.

\section{Comparison with flume-experiments data}

The experimental apparatus used to reproduce the formation of rhomboid erosion patterns is presented in details in Devauchelle et al. (2008). The following section aims only at a brief and general description.

\subsection{The experimental set-up}

The experiments of Devauchelle et al. (2008) were performed in a small channel of length $240 \mathrm{~cm}$ and width $9.6 \mathrm{~cm}$. The granular bed is made of silica beads of mean diameter $75 \mu \mathrm{m}$. The slope $S$ in the flow direction $x$ can be freely varied, as well as the water outflow. The flow depth never exceeds a few millimeters. The special features of this experiment are its small size, as well as the use of a mixture of glucose and water (the mixture viscosity $\nu$ varies between $10^{-6} \mathrm{~m}^{2} \mathrm{~s}^{-1}$ and $5.610^{-6} \mathrm{~m}^{2} \mathrm{~s}^{-1}$ ). These two characteristics yields flows at low Reynolds number (generally a few tens, and always less than $500)$.

The surface tension $\gamma$ of the water-glucose mixture was not measured during the experiments (the mass ratio of glucose to water was varied up to 50\%). However, the influence of glucose on surface tension is fairly moderate (the surface tension of a $17 \%$ mixture increases of less than $2 \%$, and the surface tension of a $55 \%$ water-sucrose mixture increases of about 5\%, see Docoslis et al. (2000)). In the following, we will thus consider that the surface tension of the mixture can be approached by the pure water value, that is $7410^{-3} \mathrm{Nm}^{-1}$. 

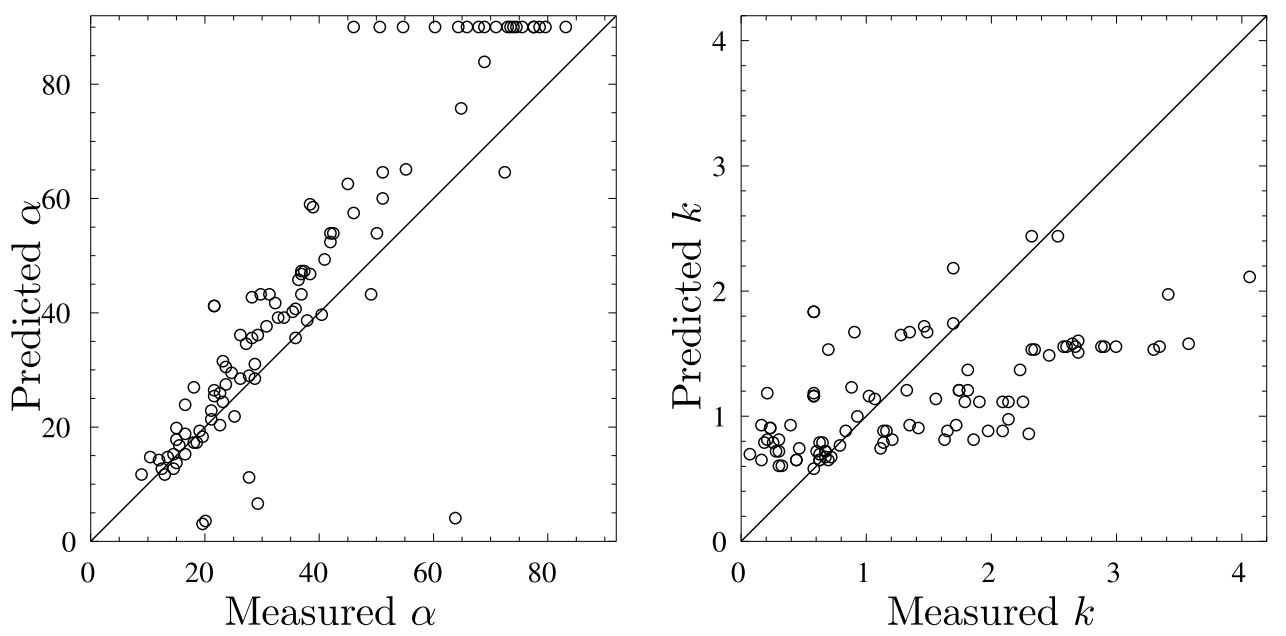

Figure 6. Comparison between the three-dimensional model of the present paper, and the experimental results of Devauchelle et al. (2008). The comparison concerns the geometrical characteristics of the rhomboid pattern: opening angle $\alpha=\pi / 2-\varphi$ (in degrees) and non-dimensional wave number $k$. The sediment transport law employed here is a generalization in three dimensions of the model initially proposed by Charru \& Hinch (2006). We used the flow parameters measured during the experiments for the stability analysis. The parameters of the erosion law are provided by Charru (2006). No additional free parameter was required for our analysis. On both plots, the solid line corresponds to the one-to-one relationship.

\subsection{Experimental patterns and stability results}

For each experimental run, the parameters $\mathrm{Fr}, \mathrm{Bo}$ and $\mathrm{Sh}$ can be determined from the measured quantities (slope $S$, water discharge and viscosity), under the assumption of a Nußelt base sate. The associated dispersion relation is then determined as described in section 3. In theory, one could just pick up the absolute maximum of the growth rate, and compare its characteristics with measurements. However, in the present case, the experimental procedure was designed to focus on rhomboid patterns. Devauchelle et al. (2008) consequently measured only the angle and wavelength of rhomboid patterns, even when ripples co-existed. Accordingly, the procedure here employed consisted in the following steps:

(a) Computing numerically the dispersion relation for each experimental run (see figure 3);

(b) Determining manually the rough position of the maximum corresponding to the bar instability;

(c) Refining numerically the position of this maximum.

Only seldom where longitudinal striations and bar instability difficult to distinguish on the dispersion-relation plot. Taking into account the relative heights of the maxima when comparing to measurements is not straightforward, since one should either measure the growth rate, or be certain that all modes are equally excited during the preparation of the sediment bed. Both would be challenging experimental problems.

The experimental results are compared to our stability analysis on figure 6 . The opening angle prediction is rather correct, given the measurement precision (about $10^{\circ}$, the pattern being usually fainter than it appears on figure 1). The wave number prediction is less accurate, but gives the correct order of magnitude and tendency. Both results are far more realistic than the ones obtained with the Saint-Venant approximation (Devauchelle et al. 2008). The discrepancy between observed and theoretical angles at extreme values 
indicates the high sensitivity of the stability analysis with respect to both the experimental parameters and the transport law parameters. When the bar maximum gets close to another local maximum (the one corresponding to longitudinal striations at low $\alpha$, the ripples one at high $\alpha$ ), a slight change in the parameter values can induce its absorption into this other maximum.

\section{Discussion and conclusion}

The present paper proposes a natural framework to model the stability of laminar films with respect to sediment transport by bedload. It is shown that a generalization in three dimensions of the transport law proposed by Charru \& Hinch (2006) and Charru (2006) can account for the beach rhomboid patterns initiation. It is thus fair to say that the diamond-shaped structures commonly observed on beaches are the laminar counterparts of alternate bars in rivers.

In return, since the rhomboid pattern is easily produced experimentally, it can be used as a test case for sediment transport models. Their three-dimensional structures add one more constraint on the bedload model (the opening angle) as compared to ripples. Transport laws are a key issue in geomorphology, and the bed slope influence on bedload remains a modeling challenge, especially in the transverse direction.

The sediment transport law successfully used here was derived from a grains balance equation written for the bedload layer. This may indicates that laws for turbulent flows similarly derived could improve our understanding of rivers bedforms generation. This is the method used by Parker et al. (2003) to develop a new bedload transport model. To the heavy experiments conduced by Francalanci \& Solari (2006) in order to evaluate the latter model, one could associate rhomboid pattern experiments, since these structures can also develop in turbulent flows (Ikeda 1983; Morton 1978). Interpreting such an experiment would require a tree-dimensional stability analysis, with a turbulent flow and a free surface, similar to the one proposed by Besio et al. (2006) for marine sand banks.

A next step toward the understanding of the rhomboid beach pattern would be to study its finite-amplitude behaviour, and particularly its saturation. Such an effort would allow to draw two new constraints on the sediment transport laws, namely the bedforms amplitude and velocity.

It is our pleasure to thank François Métivier, Clément Narteau, Stéphane Zaleski, Philippe Claudin and Bruno Andreotti for fruitful discussions. We also wish to express appreciation to Antonio Vieira and Yves Gamblin for the building of the experimental set-up.

\section{REFERENCES}

Allen, J. R. L. 1982 Sedimentary Structures-Their Character and Physical Basis, Vol. II. Developments in Sedimentology, 30b. Elsevier, Amsterdam 663, 395-405.

Andreotti, B., Claudin, P. \& Douady, S. 2002 Selection of dune shapes and velocities Part 2: A two-dimensional modelling. The European Physical Journal B-Condensed Matter 28 (3), 341-352.

Bagnold, R. A. 1977 Bed load transport by natural rivers. Water Resources Research 13 (2), 303-312.

Besio, G., Blondeaux, P. \& Vittori, G. 2006 On the formation of sand waves and sand banks. J. Fluid Mech. 557, 1-27.

Blondeaux, P. \& Seminara, G. 1985 A unified bar-bend theory of river meanders. J. Fluid Mech. 157. 
Callander, R. A. 1969 Instability and river channels. J. Fluid Mech. 36, 465-480.

Chang, H. Y. \& Simons, D. B. 1970 The bed configuration of straight sand beds when flow is nearly critical. J. Fluid Mech. 42, 491-495.

Charru, F. 2006 Selection of the ripple length on a granular bed sheared by a liquid flow. Physics of Fluids 18, 121508.

Charru, F. \& Hinch, E. J. 2006 Ripple formation on a particle bed sheared by a viscous liquid. Part 1. Steady flow. J. Fluid Mech. 550, 111-121.

Charru, F., Mouilleron, H. \& Eiff, O. 2004 Erosion and deposition of particles on a bed sheared by a viscous flow. J. Fluid Mech. 519, 55-80.

Charru, F. \& Mouilleron-Arnould, H. 2002 Instability of a bed of particles sheared by a viscous flow. J. Fluid Mech. 452, 303-323.

Coleman, S. E. \& Eling, B. 2000 Sand wavelets in laminar open-channel flows. J. of Hydraulic Research/Journal de Recherches Hydraulique 38 (5), 331-338.

Colombini, M. 2004 Revisiting the linear theory of sand dune formation. J. Fluid Mech. 502, $1-16$.

Colombini, M. \& PArker, G. 1995 Longitudinal streaks. J. Fluid Mech. 304.

Colombini, M. \& Stocchino, A. 2005 Coupling or decoupling bed and flow dynamics: Fast and slow sediment waves at high Froude numbers. Physics of Fluids 17, 036602.

Defina, A. 2003 Numerical experiments on bar growth. Water Resour. Res 39 (4), 1092.

Devauchelle, O., Josserand, C., Lagrée, P. Y. \& Zaleski, S. 2007 Morphodynamic modeling of erodible laminar channels. Physical Review E 76 (5), 56318.

Devauchelle, O., Malverti, L., Lajeunesse, É., Josserand, C., Lagrée, P. Y. \& MÉTIVIER, F. 2008 Rhomboid beach pattern: a benchmark for shallow water geomorphology. J. of Geophysical Research Submitted.

Docoslis, A., Giese, R. F. \& VAn Oss, C. J. 2000 Influence of the water-air interface on the apparent surface tension of aqueous solutions of hydrophilic solutes. Colloids and Surfaces B: Biointerfaces 19 (2), 147-162.

Elbelrhiti, H., Claudin, P. \& Andreotti, B. 2005 Field evidence for surface-wave-induced instability of sand dunes. Nature 437 (7059), 720-723.

Exner, F. M. 1925 Über die Wechselwirkung zwischen Wasser und Geschiebe in Flüßen. Sitzenberichte Akad. Wiss. Wien 165 (3-4), 165-203.

Francalanci, S. \& Solari, L. 2006 A particle tracking technique to study gravitational effects on bedload transport. In River, Coastal, and Estuarine Morphodynamics: RCEM 2005: Proceedings of the 4th IAHR Symposium on River Coastal and Estuarine Morphodynamics, 4-7 October 2005, Urbana, Illinois, USA. Taylor \& Francis.

HALl, P. 2006 Nonlinear Evolution Equations and the Braiding of Weakly Transporting Flows Over Gravel Beds. Studies in Applied Mathematics 117 (1), 27-69.

Hersen, P. 2004 On the crescentic shape of barchan dunes. The European Physical Journal B-Condensed Matter and Complex Systems 37 (4), 507-514.

IKEDA, H. 1983 Experiments on bedload transport, bed forms, and sedimentary structures using fine gravel in the 4-meter-wide flume. Environmental Research Center papers 2, 1-78.

IKedA, S., PARker, G. \& SAWAI, K. 1981 Bend theory of river meanders. Part 1. Linear development. J. Fluid Mech. 112, 363-377.

JACKSON, R.G. 2006 Sedimentological and fluid-dynamic implications of the turbulent bursting phenomenon in geophysical flows. J. Fluid Mech. Digital Archive 77 (03), 531-560.

KapitZA, P. L. 1948 Wave Motion of a Thin Layer of a Viscous Liquid-Part I. Zh. eksp. teor. Fiz 18, 3 .

KArCZ, I. \& Kersey, D. 1980 Experimental Study of Free-Surface Flow Instability and Bedforms in Shallow Flows. Sedimentary Geology 27 (4), 263-300.

Kennedy, J. F. 1963 The mechanics of dunes and antidunes in alluvial channels. J. Fluid Mech $16(4), 521-544$.

KnaApen, M. A. F. \& Hulscher, S. 2003 Use of a genetic algorithm to improve predictions of alternate bar dynamics. Water Resources Research 39 (9), 1231.

Kouakou, K. K. J. \& Lagrée, P. Y. 2005 Stability of an erodible bed in various shear flows. The European Physical Journal B-Condensed Matter 47 (1), 115-125. 
Kuru, W. C., Leighton, D. T. \& MCCready, M. J. 1995 Formation of waves on a horizontal erodible bed of particles. International J. of Multiphase Flow 21 (6), 1123-1140.

LAGrée, P. Y. 2000 Erosion and sedimentation of a bump in fluvial flow. Comptes Rendus de l'Académie des Sciences Séries IIB Mechanics 328 (12), 869-874.

LagréE, P. Y. 2003 A triple deck model of ripple formation and evolution. Physics of Fluids 15, 2355 .

Lajeunesse, E., Malverti, L., Lancien, P., Armstrong, L., Metivier, F., Coleman, S., Smith, C., Davies, T., Cantelli, A. \& Parker, G. 2008 Fluvial and subaqueous morphodynamics of laminar and near-laminar flows. Sedimentology Submitted.

LANGLOIS, V. 2005 Instabilité d'un lit granulaire cisaillé par un écoulement fluide. PhD thesis, Rennes 1 University, in French.

Langlois, V. \& Valance, A. 2005 Formation of Two-Dimensional Sand Ripples under Laminar Shear Flow. Physical Review Letters 94 (24), 248001.

Lin, S. P. \& ChEn, J. N. 1997 Elimination of three-dimensional waves in a film flow. Physics of Fluids 9, 3926.

Morton, R. A. 1978 Large-scale rhomboid bed forms and sedimentary structures associated with hurricane washover. Sedimentology 25, 183-204.

Parker, G., Seminara, G. \& Solari, L. 2003 Bed load at low Shields stress on arbitrarily sloping beds: Alternative entrainment formulation. Water Resources Research 39 (7), 1183.

Reynolds, A. J. 1965 Waves on the erodible bed of an open channel. J. Fluid Mech. 22, $113-133$.

Sauermann, G., Kroy, K. \& Herrmann, H.J. 2001 Continuum saltation model for sand dunes. Physical Review E 64 (3), 31305.

Shields, A. 1936 Anwendung der Aehnlichkeitsmechanik und der Turbulenzforschung auf die Geschiebebewegung. Preussische Versuchsanstalt für Wasserbau und Schiffbau.

SinGH, I. B. 1969 Primary sedimentary structures in Precambrian quartzites of Telemark, southern Norway and their environmental significance. Nor. Geol. Tidsskr 49, 1-31.

Thompson, W. O. 1949 Lyons sandstone of Colorado Front Range. AAPG Bulletin 33 (1), $52-72$.

Valance, A. \& Langlois, V. 2005 Ripple formation over a sand bed submitted to a laminar shear flow. The European Physical Journal B-Condensed Matter 43 (2), 283-294.

Valance, A. \& Rioual, F. 1999 A nonlinear model for aeolian sand ripples. The European Physical Journal B-Condensed Matter 10 (3), 543-548.

Williamson, W. C. 1887 On Some Undescribed Tracks of Invertebrate Animals from the Yoredale Rocks, and on some Inorganic Phenomena, Produced on Tidal Shores, Simulating Plant Remains. Manchester Literary and Philosophical Society, Memoirs and Proceedings, ser 3 (10), 19-29.

Woodford, A. O. 1935 Rhomboid ripple mark. Amer. J. Science, 5th series 29, 518-525.

Yıн, C. S. 1963 Stability of Liquid Flow down an Inclined Plane. Physics of Fluids 6. 